\title{
OFDM SIGNAL RESEARCH WITH VARIED SUBCARRIERS NUMBER
}

\author{
Leonid Uryvsky ${ }^{1}$, Serhii Osypchuk ${ }^{2}$ \\ Institute of Telecommunication Systems \\ National Technical University of Ukraine "Kyiv Polytechnic Institute" \\ Kyiv, Industrialny Street, 2, Ukraine, 03057 \\ E-mails: ${ }^{1}$ leonid_uic@ukr.net; ${ }^{2}$ serhii.osypchuk@ieee.org \\ ORCID: ${ }^{10000-0002-4073-9681 ;{ }^{2} 0000-0002-6174-2986}$
}

The orthogonal frequency division multiplexing (OFDM) aggregate signal energy characteristics are studied based on the OFDM signal simulation model. The information reliability of OFDM signal is explored for different sets of OFDM subcarrier numbers and varied multiposition keying types (MPK) on OFDM subcarriers. The conditions for optimal gain factor for OFDM signal are described analytically based on the simulation modeling results. The effect and value from using an optimal gain factor for OFDM signal is shown in this paper.

Keywords: OFDM, simulation, subcarriers number, multiposition keying, BER, optimal gain factor

\section{Introduction}

Orthogonal frequency division multiplexing named OFDM (Chang, 1966) is a widely used technique in modern wireless, wired and optical telecommunication systems (Prasad, 2004 and Arunabha, 2004).

OFDM is a technique where the below steps are realized sequentially:

- division of input bits stream from a source to $N_{\text {OFDM }}$ substreams with an equal data rates for each substream where every substream rate is $N_{\text {OFDM }}$ times smaller than an input stream rate;

- each substream modulation with a specific type of multiposition keying (MPK);

- aggregate shaping from all the subcarriers with the data rate that is equal to initial stream rate;

- transmission of an aggregate OFDM signal into communication channel.

The following advantages of OFDM technology are significant: high efficiency of frequency band utilization, high resistance to signal fading in communication channel, high robustness to the impulse noise influence. At the same time, the next disadvantages of OFDM can be highlighted: low energy efficiency due to high Peak-to-Average Power Ratio (PAPR) value, high sensitivity to frequency offsets in case of Doppler effect (Arun, 2012).

The method for assessing the OFDM group signal energy parameters is described in (Uryvsky, 2013). This method uses a predefined OFDM signal subcarriers number and specific MPK type for OFDM subcarriers. It is shown in (Uryvsky, 2013) that it is possible and reasonable to use an amplification factor for the aggregate OFDM-signal for the purpose of improving the signal energy and keeping the amplification linear condition in the applicable limits. Thus, usage of the method (Uryvsky, 2013) improves the OFDM signal energy and keeps the non-linear distortions on an accepted level during OFDM signal amplification process; herewith the information reliability on the receiver side improves.

The OFDM signal is not studied in (Uryvsky, 2013) for a behavior of aggregate signal characteristics in dependence on OFDM subcarriers number and different types of MPK. This is a topical scientific and practical task; the results of this research are presented in the current paper.

\section{Problem statement}

The objective of this work is a definition of OFDM subcarriers number $N_{\text {OFDM }}$ and type of subcarriers MPK influence on parameters and factors of aggregate OFDM signal: 
- optimal voltage amplification factor $K_{\mathrm{U} \_ \text {OPT }}=f\left(N_{\text {OFDM }}, k\right)$, where $k=\log _{2} M$ - number of bits per a symbol of MPK, $M$ - number of points in MPK constellation;

- optimal power amplification factor $K_{\mathrm{P} \_ \text {OPT }}=f\left(N_{\text {OFDM }}, k\right)$;

- energy parameter $h_{\mathrm{REC}}^{2}=f\left(N_{\mathrm{OFDM}}, k\right)$ that shows the rate of elementary signal energy to white noise spectral density;

- $\operatorname{symbol}$ error rate (SER) for MPK $p_{\mathrm{S}}=f\left(N_{\text {OFDM }}, k\right)$;

- bit error rate (BER) for MPK $p_{\mathrm{b}}=f\left(N_{\text {OFDM }}, k\right)$;

- characteristic that shows ratio of optimal subcarriers power amplification factor to subcarriers number (1); that's a metric for efficiency usage of power resource by OFDM in comparison with a single carrier (SC) transmission:

$\frac{K_{\text {P_OPT }}}{N_{\text {OFDM }}}=f\left(N_{\text {OFDM }}, k\right)$.

\section{Technique for researching the OFDM signal characteristics}

Statistical properties of aggregate OFDM signal have a high PAPR value, and this circumstance significantly decreases the efficiency of energy resource usage by OFDM technique. A critical objective is the task of keeping an aggregate OFDM signal level in the bounds of linear section in amplification characteristic of transmitter.

An amplification of aggregate OFDM signal in transmitter with some voltage amplification factor $K_{\mathrm{U}}$ increases an average level of transmitted and received OFDM signal respectively. Such amplification promotes an improvement of information reliability on the receiver side, but it can exceed with some probability the linear section of amplification characteristic in transmitter. An optimal voltage amplification factor $K_{\mathrm{U}_{-} \mathrm{OPT}}$ is supposed when the condition of values equality is met: nonlinear distortions probability $p_{\mathrm{NL}}$ in the transmitter's amplifier and symbol error probability $p_{\mathrm{S}}$ for a specific MPK in the receiver:

$p_{\mathrm{NL}}=p_{\mathrm{S}}$.

In other words, the optimum voltage gain factor is defined by the next functional:

$K_{\mathrm{U} \_ \text {OPT }}=f\left(\left\{p_{\mathrm{S}}, p_{\mathrm{NL}}\right\}=f\left(h_{\mathrm{REC}}^{2}, k, N_{\mathrm{OFDM}}\right)\right)$.

An example of the functional (3) that is dedicated to OFDM signal energy characteristics research is shown on the Fig. 1.

The value of BER $p_{\mathrm{b}_{-} 0}=10^{-6}$ means such a level of information reliability that is reached on the receiver side when SC transmission is used with MPK QAM-64 and channel energy parameter in the receiver point is $h_{\mathrm{REC}}^{2}$ (Uryvsky, 2013):

$h_{\mathrm{REC}}^{2}=\frac{P_{\mathrm{S}} T_{\mathrm{S}}}{\mathrm{N}_{0}}$,

where $P_{\mathrm{S}}$ is a power of signal at the receiving point; $T_{\mathrm{S}}=1 / V_{\mathrm{S}}-$ length of signal, $V_{\mathrm{S}}-$ symbols data rate; $\mathrm{N}_{0}-$ spectral density of white noise. The model of channel with AWGN is taken in this research due to couple of things: 1) the research is dedicated to the physical properties of OFDM signal, not for channel models; 2) energy parameter $h_{\mathrm{REC}}^{2}$ always can be recalculated into the respective equivalent $\hat{h}_{\mathrm{REC}}^{2}$ parameter for other channel models like multipath model etc.; 3) improving the OFDM signal energy level is useful for all the channel types and the idea is general and independent of the channel model. 


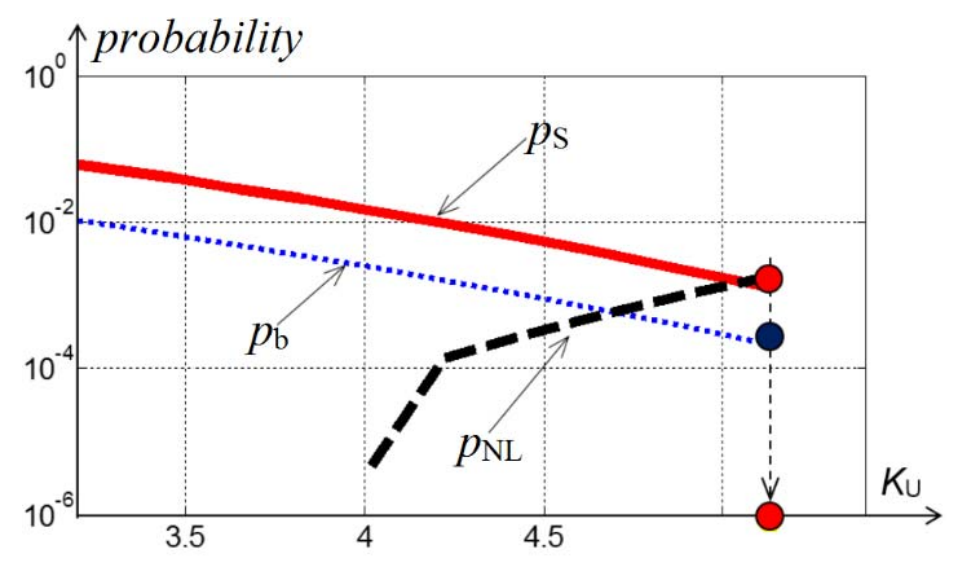

Figure 1. Achieving the condition $p_{\mathrm{NL}}=p_{\mathrm{S}}=1.1 \cdot 10^{-3}$ when $K_{\mathrm{U} \_\mathrm{OPT}}=5.1$ for aggregated OFDM signal; achieved BER at this point: $p_{\mathrm{b}}=2 \cdot 10^{-4}$. Initial parameters: $N_{\mathrm{OFDM}}=10$; subcarriers MPK: QAM-64; the required BER that could be reached when SC transmission is used with QAM-64: $p_{\mathrm{b}_{-} 0}=10^{-6}$

$\operatorname{SER}\left(p_{\mathrm{S}}\right)$ and $\operatorname{BER}\left(p_{\mathrm{b}}\right)$ values in the receiving point on the one hand depend on a signal energy and AWGN spectral density rate: $h_{\mathrm{REC}}^{2}$; on the other hand, SER and BER values depend on MPK type with parameter $k=\log _{2} M$ bits per a symbol. Thus, it's possible to measure SER and BER when MPK is used. At the same time, the channel productivity increases with growing $k$, but this accompanied with worsening the SER/BER levels, so a compromise needs to be found between MPK growing and required SER/BER levels in the receiving point.

At the same time, OFDM technique uses tens, hundreds or thousands subcarriers, and every subcarrier is modulated by MPK. OFDM aggregate signal level strives to zero if the number of subcarriers grows and if transmitted data have statistically random zeros and ones.

Thereby, significant OFDM PAPR value and high MPK value $M$ are the prerequisite for reasonable signal energy increasing in transmitter; important thing in this case is that the amplified signal level in transmitter should be in acceptable bounds of non-linear distortions in amplifier. The below chapters show the results of researching an optimal OFDM signal amplification value.

\section{OFDM aggregate signal parameters research as a function of subcarriers number NOFDM}

As the first step to the aggregate OFDM signal with MPK simulation modelling, the distribution of the aggregate OFDM signal is given for the cases: 1) before amplifying the aggregate OFDM signal; 2) after amplifying the aggregate OFDM signal.

Three OFDM aggregate signal amplitude distribution functions are shown on Fig. 2: before amplification (lines: \#1 and \#2), and after signal amplification with optimal gain factor $K_{\mathrm{U}_{-} \text {орт }}$ (line \#3).

This experiment shows that OFDM aggregate signal amplitude distribution function depends on MPK insignificantly, but it depends considerably on OFDM subcarriers number. On Fig. 2: the initial maximal aggregate signal voltage amplitude on the output of transmitter's amplifier is $U_{0}=10 \mathrm{~V}$ (non-linear distortions are zero here: $\left.p_{\mathrm{NL}}=0\right)$. In this case the energy parameter $h_{\mathrm{REC}}^{2}$ (4) for SC transmission transforms into the OFDM signal energy parameter $h_{\text {OFDM }}^{2}: h_{\text {OFDM }}^{2}=h_{\mathrm{REC}}^{2} / N_{\text {OFDM }}$. The next values are shown on Fig. 2: $U_{0}=10 \mathrm{~V}$, where $p_{\mathrm{NL}}=0 ; \quad U=10 \ldots 12.8 \mathrm{~V}$, where $p_{\mathrm{S}}>p_{\mathrm{NL}}>0$; $U_{\mathrm{OPT}}=12.8 \mathrm{~V}$, where $p_{\mathrm{S}}=p_{\mathrm{NL}}$ and $U_{\mathrm{OPT}}=K_{\mathrm{U} \_\mathrm{OPT}} \cdot U_{0}$. The lines \#1 and \#3 in Fig. 2 correspond to MPK signal QAM-64 ( $\left.N_{\text {OFDM }}=60\right)$, the line \#2 corresponds to MPK signal QPSK $\left(N_{\text {OFDM }}=20\right)$. 


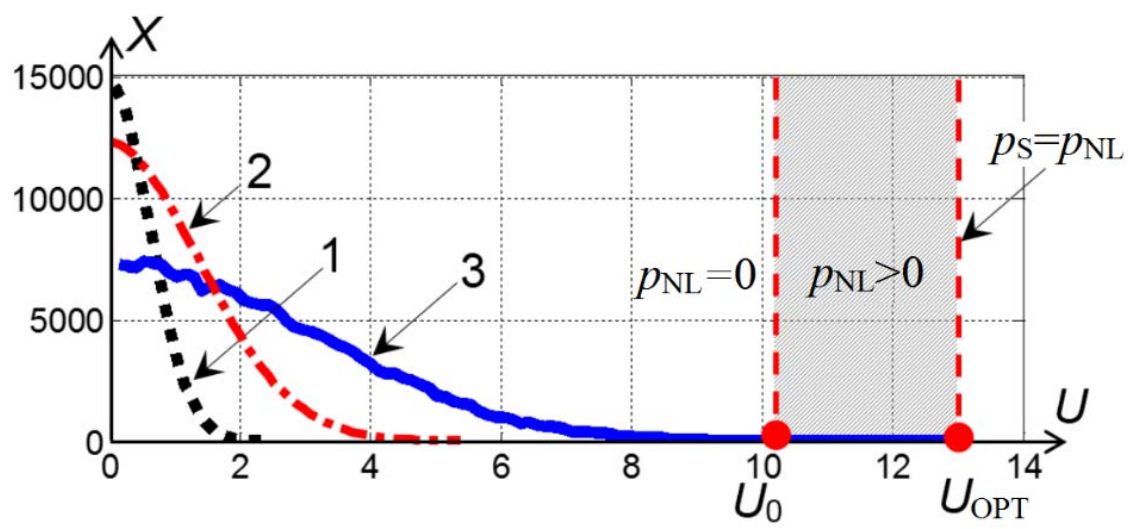

Figure 2. OFDM aggregate signal distribution functions: \#1 and \#2 - before amplification; \#3 - after amplification, $X$ axis - the number of specific OFDM aggregate signal level samples

As the statistical experiment shown in Fig. 3, the distribution function of OFDM signal momentary level values in approximation comes nearer to the truncated normal distribution law together with growing the OFDM subcarriers number:

$f(x)=\frac{1}{\sigma \sqrt{2 \pi}} \exp \left(-\frac{(x-\mu)^{2}}{2 \sigma^{2}}\right)$,

where $\mu=0$ is a mathematical expectation of distribution density for function $f(x), \sigma^{2}$ is a distribution dispersion, $\sigma$ - mean-square deviation.

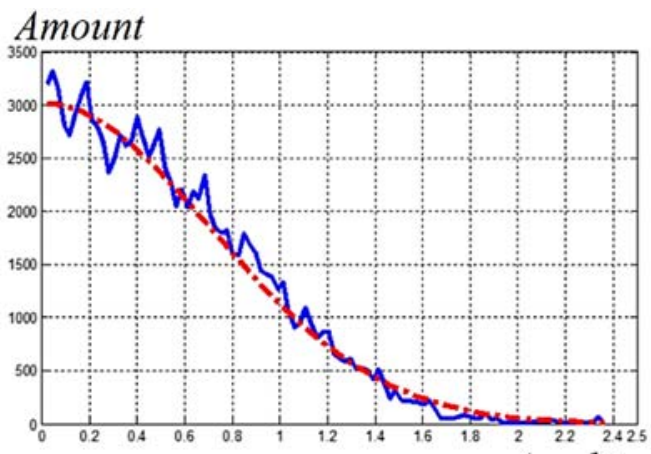

a)

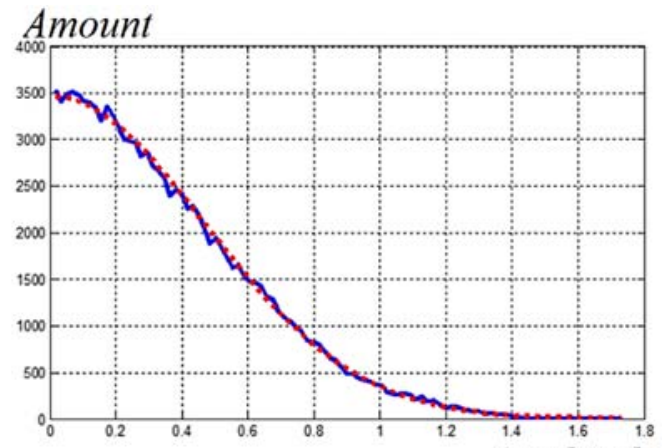

b)
Amplitude

Figure 3. Approximation of OFDM aggregate signal distribution function in the form of truncated normal distribution law: a) $N_{\mathrm{OFDM}}=16$, QAM-16; б) $N_{\mathrm{OFDM}}=32$, QAM-64. An approximation for b) is completed with the precise level 0.9898 by the chi-square criterion

The dependencies of initial mean-square deviation value $\sigma$ before OFDM signal amplification and after OFDM signal gain as a function of subcarriers number $N_{\text {OFDM }}$ are shown in Fig. 4.

As the research has demonstrated, a mean-square value $\sigma$ of distribution (5) depends on the OFDM subcarriers number $N_{\text {OFDM }}$ before amplification (6a), and it doesn't depend on subcarriers number $N_{\text {OFDM }}$ after amplification (6b):

$\sigma=\left(0,1558 k_{\mathrm{QAM}}^{2}-1,9053 k_{\mathrm{QAM}}+10,629\right) \cdot N_{\mathrm{OFDM}}^{-0.504}$,

$\sigma_{K_{\mathrm{U}}}=0,0294 k_{\mathrm{QAM}}^{2}-0,3875 k_{\mathrm{QAM}}+4,58$,

where $k_{\mathrm{QAM}}=2$ for QPSK, $k_{\mathrm{QAM}}=4$ for QAM-16 and $k_{\mathrm{QAM}}=6$ for QAM- 64 . 


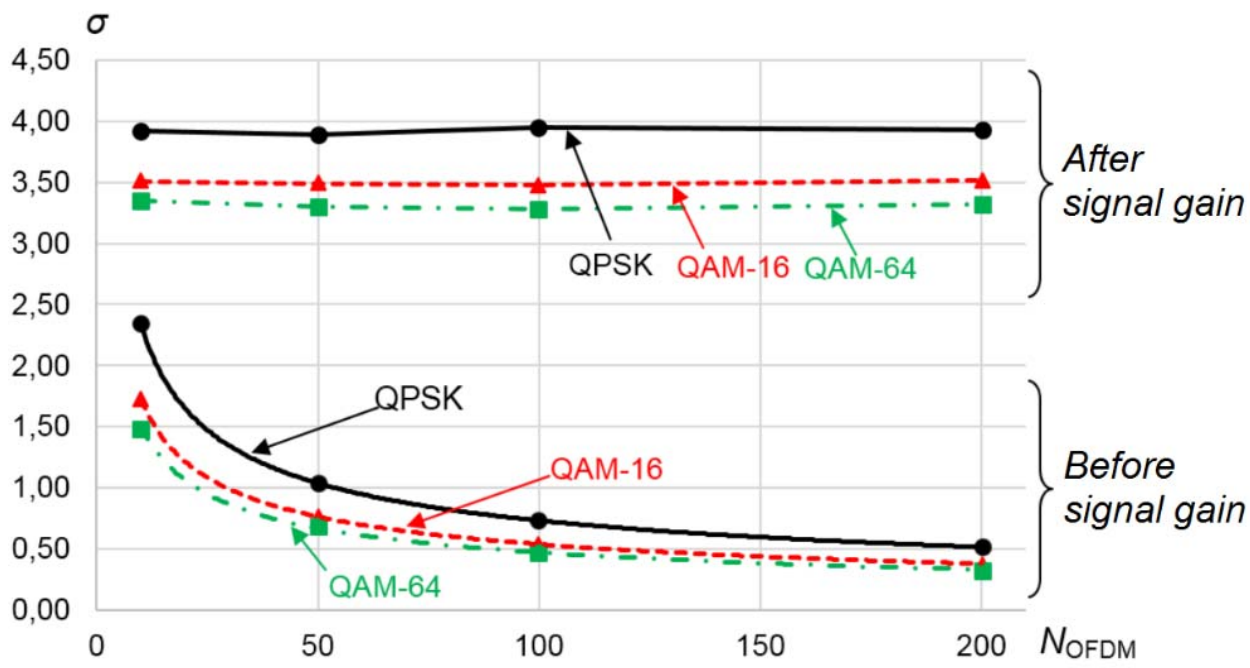

Figure 4. The dependency of mean-square deviation $\sigma$ from OFDM subcarriers number $N_{\text {OFDM }}$

A hypothesis that the OFDM aggregate signal amplitudes distribution function follows the normal distribution law with parameter $\sigma(6 \mathrm{a}, 6 \mathrm{~b})$ is confirmed by chi-square criterion with the precise level 0.9898 .

The normalization condition for normal distribution law $f(x)$ (5) has the form (7) before the OFDM signal amplification:

$\frac{1}{\int_{0}^{U_{0}} f(x) d x} \int_{0}^{U_{0}} f(x) d x=1$,

and it means that a distribution function truncation is limited in the linear part of amplifier gain characteristic $\left(0 \ldots U_{0}\right)$; for this case the distribution (5) is used with mean-square value $\sigma$ (6a).

After OFDM signal amplification with gain factor $K_{\mathrm{U}}$, maximal possible amplitude of the OFDM aggregate signal increases up to level $U_{\mathrm{MAX}}=K_{\mathrm{U}} \cdot U_{0}$, and parameter $\sigma_{K_{\mathrm{U}}}$ of distribution (5) increases proportionally up to level $\sigma_{K_{\mathrm{U}}}\left(N_{\text {OFDM }}\right)=\sigma_{K_{\mathrm{U}}}\left(N_{\text {OFDM }}\right) \cdot K_{\mathrm{U}}\left(N_{\text {OFDM }}\right)$ and is defined by the expression (6b). As we can see, $\sigma_{K_{\mathrm{U}}}$ in (6b) doesn't depend on the subcarriers number $N_{\text {OFDM }}$ (Fig. 4). Thereafter, new distribution density equation takes on form:

$$
f_{\mathrm{U}}(x)=\frac{1}{\sigma_{K_{\mathrm{U}}} \sqrt{2 \pi}} \cdot \exp \left(-\frac{x^{2}}{2 \cdot \sigma_{K_{\mathrm{U}}}^{2}}\right) .
$$

The probability of situation when an amplified OFDM aggregate signal exceeds an amplifiers' linear gain characteristic takes on the form:

$$
\frac{1}{\int_{0}^{K_{\mathrm{U}} U_{0}} f_{\mathrm{U}}(x) d x} \int_{0}^{U_{0}} f_{\mathrm{U}}(x) d x<1,
$$

Consequently, the condition for optimal gain factor $K_{\mathrm{U}_{-} \mathrm{OPT}}$ in concordance with expression (2) is realized based on the solving an integral equation: 


$$
\frac{1}{\int_{0}^{K_{\mathrm{U}} \cdot U_{0}} f_{\mathrm{U}}(x) d x} \int_{U_{0}}^{K_{\mathrm{U}} \cdot U_{0}} f_{\mathrm{U}}(x) d x=p_{\mathrm{S}},
$$

where the form of function $f_{\mathrm{U}}(x)$ is defined by expression (8) with parameter $\sigma_{\mathrm{U}}(6 \mathrm{~b})$, when values $U_{0}$ and $p_{\mathrm{S}}$ are defined, and the value $K_{\mathrm{U}}=K_{\mathrm{U}_{\mathrm{OOPT}}}$ is sought-for.

On the second phase of modelling the relationship is set up between the OFDM aggregate signal optimal gain factor $K_{\mathrm{U} \_ \text {опт }}$ and OFDM subcarriers number $N_{\text {ОFDM }}$.

The research results of optimal OFDM signal gain $K_{\mathrm{U}_{-} \text {OPт }}$ for OFDM subcarriers number $N_{\text {OFDM }}=10 \ldots 250$ are shown in Fig. 5 for initial BER level $p_{\mathrm{b}_{-} 0}=10^{-6}$ and MPK types QPSK, QAM16, QAM-64 when conditions (2) and (10) are complied. Parameters of frequency band and white noise level are constant for all experiments. Mentioning the initial BER level $p_{\mathrm{b}_{\_} 0}=10^{-6}$ refers exceptionally to the mode of SC transmission when BER $p_{\mathrm{b}_{-} 0}=10^{-6}$ is reached on the receiver side and $K_{\mathrm{U}_{-} \text {опт }}=1$ for $\mathrm{SC}$ transmission. In this case during a changeover of transmission modes from $\mathrm{SC}$ transmission to OFDM transmission, the communication channel parameters are constant (white noise level $\mathrm{N}_{0}$ ).

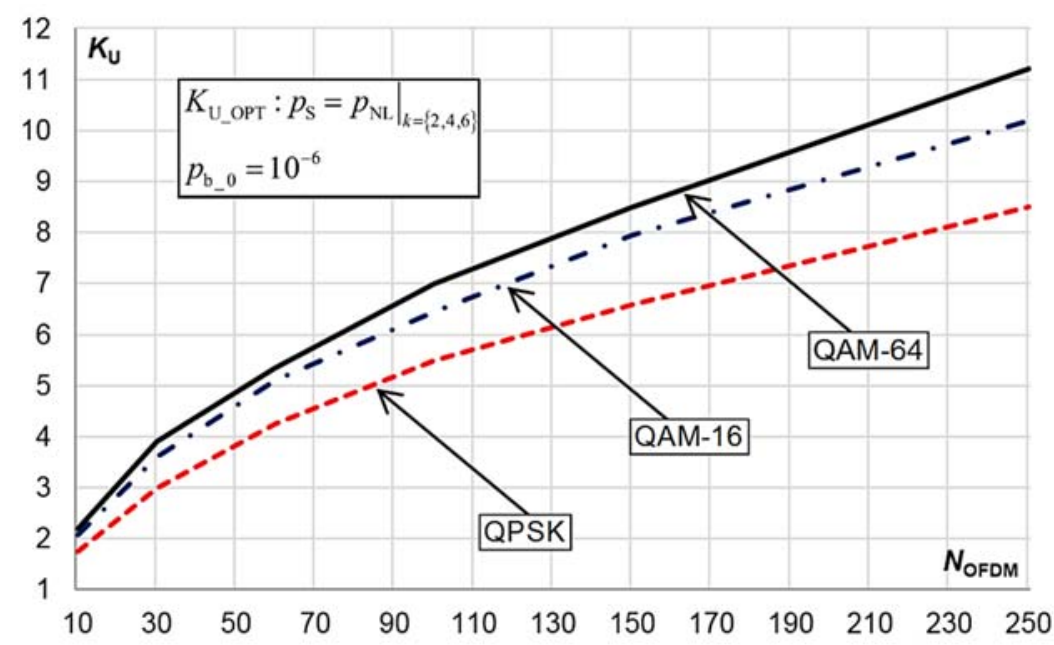

Figure 5. OFDM aggregate signal optimal gain factor $K_{\mathrm{U}_{-} \mathrm{OPT}}$ for different MPK types

Optimal voltage $K_{\mathrm{U}_{-} \mathrm{OPT}}$ and power $K_{\mathrm{P}_{-} \mathrm{OPT}}$ gain factors are connected by the next expression:

$$
K_{\mathrm{P}_{-} \mathrm{OPT}}=\left(K_{\mathrm{U}_{-} \mathrm{OPT}}\right)^{2} \text {. }
$$

Optimal OFDM aggregate signal power gain factor $K_{\mathrm{P}_{-} \mathrm{OPT}}$ is shown in Fig. 6.

The dependency of communication channel energy parameter $h_{\mathrm{OFDM}}^{2}$ in the receiving point after optimal amplification the aggregate OFDM signal with gain factor $K_{\mathrm{P} \_ \text {OPT }}$ is shown in Fig. 7.

The new result in Fig. 7 that was not introduced earlier anywhere is the circumstance that with using of optimal amplification factor $K_{\mathrm{P}_{\mathrm{OPT}}}$ for the OFDM aggregate signal, with compliance of conditions (2) and (3), the communication channel energy parameter $h_{\mathrm{OFDM}}^{2}$ at the same receiving point is actually invariable at the time when different OFDM subcarriers number $N_{\text {OFDM }}$ is used for pre-defined MPK type for OFDM subcarriers. 


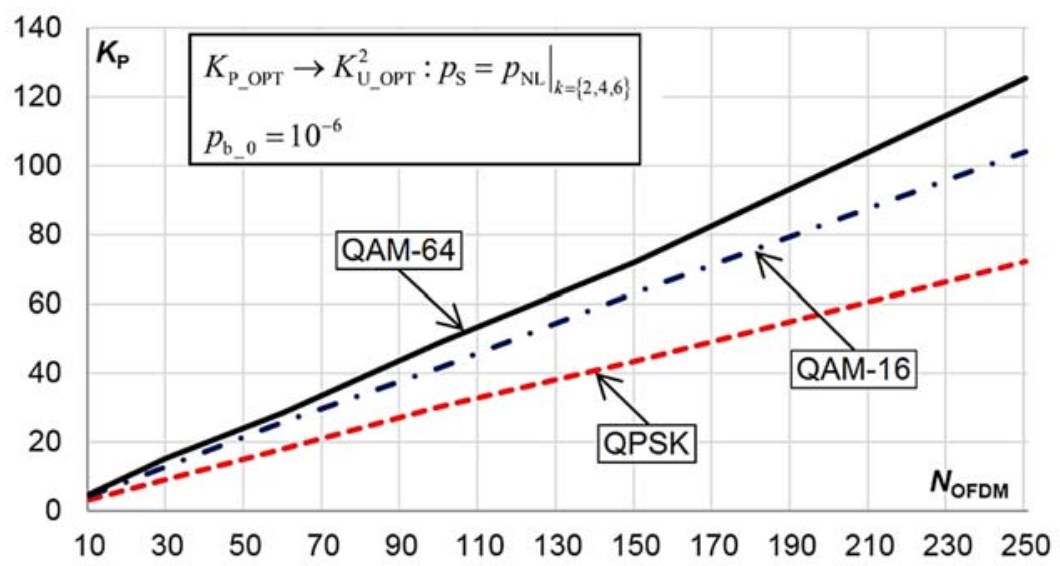

Figure 6. OFDM aggregate signal optimal gain factor $K_{\mathrm{P} \_ \text {OPT }}$ for MPK types: QPSK, QAM-16, QAM-64

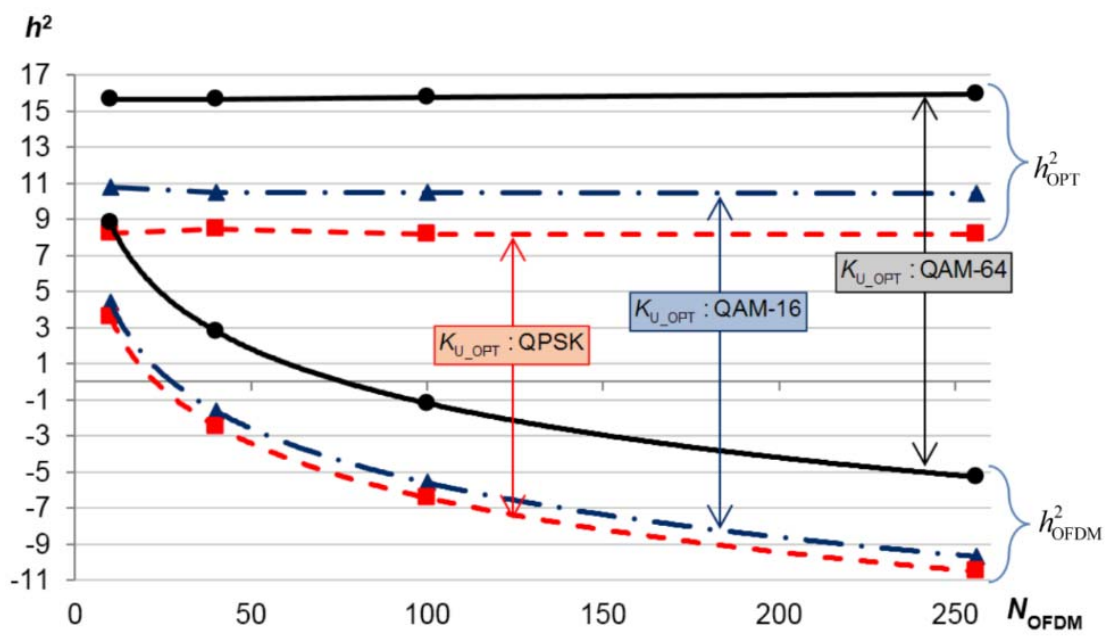

Figure 7. Channel energy parameter $h_{\mathrm{OFDM}}^{2}=f\left(N_{\mathrm{OFDM}}, k, K_{\mathrm{U} \_\mathrm{OPT}}\right): p_{\mathrm{S}}=\left.p_{\mathrm{NL}}\right|_{k=\log _{2} M=\{2,4,6\}} ; p_{\mathrm{b}_{\_} 0}=10^{-6}$

As a result, an expected corollary is shown in Fig. 8 for SER value in a receiving point; SER value is invariable too when different OFDM subcarriers numbers are used and $K_{\mathrm{P} \text { OPT }}$ is used in compliance with the condition (2).

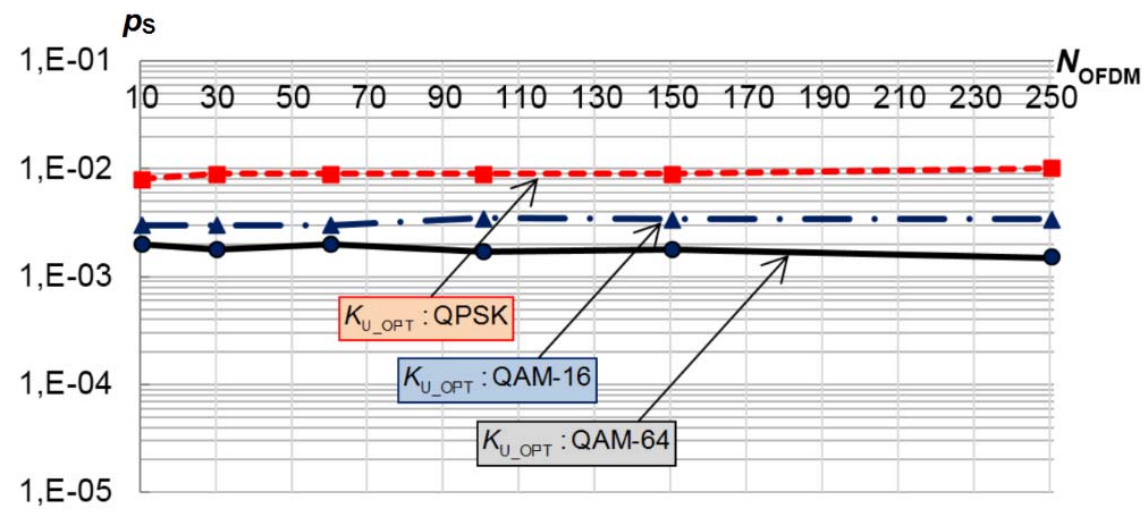

Figure 8. SER as a function of $N_{\mathrm{OFDM}}$ with $K_{\mathrm{U} \_ \text {OPT }}$ using accordingly with the condition $p_{\mathrm{NL}}=p_{\mathrm{S}}$ :

$$
p_{\mathrm{S}}=f\left(N_{\mathrm{OFDM}}, k, K_{\mathrm{U} \_ \text {OPT }}\right): p_{\mathrm{NL}}=\left.p_{\mathrm{S}}\right|_{k=\log _{2} M=\{2,4,6\}} ; p_{\mathrm{b}_{-} 0}=10^{-6}
$$


A similar tendency for BER level is noted like BER invariance in the same receiving point for whatever OFDM subcarriers number and applying a $K_{\text {Р ОРт }}$ value for aggregate OFDM signal in compliance with the condition (2) (Fig. 9).

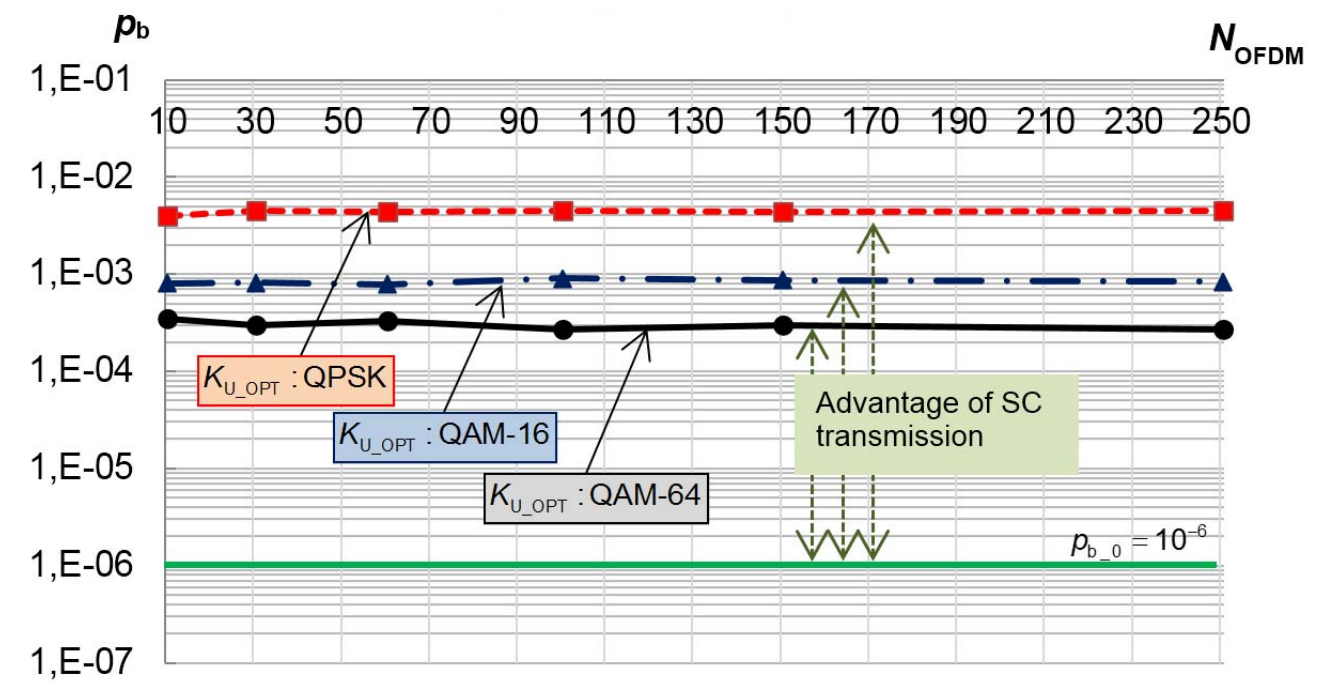

Figure 9. BER as a function of $N_{\mathrm{OFDM}}$ with $K_{\mathrm{U} \_ \text {OPT }}$ using accordingly with the condition $p_{\mathrm{NL}}=p_{\mathrm{S}}$ :

$$
p_{\mathrm{b}}=f\left(N_{\mathrm{OFDM}}, k, K_{\mathrm{U} \_ \text {OPT }}\right): p_{\mathrm{S}}=\left.p_{\mathrm{NL}}\right|_{k=\log _{2} M=\{2,4,6\}} ; p_{\mathrm{b}_{-} 0}=10^{-6}
$$

The modelling results (Fig. 7-9) are based on random OFDM signal values and statistical modelling with samples volume $n \sim 10^{5}$. A known value of $K_{\text {U_опт }}$ gives a possibility to calculate the energy parameter $h_{\mathrm{OPT}}^{2}$ (Fig. 7):

$h_{\mathrm{OFDM}}^{2}<\left(h_{\mathrm{OPT}}^{2}=K_{\mathrm{U} \_\mathrm{OPT}} \cdot \frac{h_{\mathrm{REC}}^{2}}{N_{\mathrm{OFDM}}}\right)<h_{\mathrm{REC}}^{2}$.

The results of calculation SER values $\left(p_{\mathrm{S}}\right)$ and BER values $\left(p_{\mathrm{b}}\right)$ that are shown on Fig. 8 and Fig. 9 are calculated analytically as a function of $h_{\text {OFDM }}^{2}$ (Uryvsky, 2013).

The detected effect of the above-mentioned method, that applies an optimal gain factor for the OFDM aggregate signal, provides the possibility to use this method for OFDM signal as a multistation access tool in such perspective technologies as LTE (Ghosh et al., 2004), Wi-Fi etc. This method is specifically useful when changing the amount of subscribers in a system is connected with respective changing the OFDM subcarriers number $N_{\text {OFDM }}$; together with this the energy parameter of the OFDM aggregate signal keeps on the same level (does not become worse). Consequently, resultant reliability of transmitted information is on the same level. At the same time this OFDM mode is significantly worse in words of energy utilization efficiency and information reliability if compare it with SC transmission.

\section{An optimal OFDM aggregate signal gain factor dependency from information reliability requirements research}

The dependencies of $K_{\mathrm{U} \text { OPT }}$ for the OFDM aggregate signal with subcarriers MPK type QAM-64 for different information reliability requirements (that is reached with $\mathrm{SC}$ transmission, $p_{\mathrm{b}_{-} 0}$ ) are shown in Fig. 10. As would be expected, for better information reliability in the same receiving point, it is needed to apply a bigger gain factor to the aggregate OFDM signal. Increasing the MPK factor also leads to increasing an optimal gain factor $K_{\mathrm{U} \_\mathrm{OPT}}$ as shown in Fig. 10 for QPSK and QAM-64. 


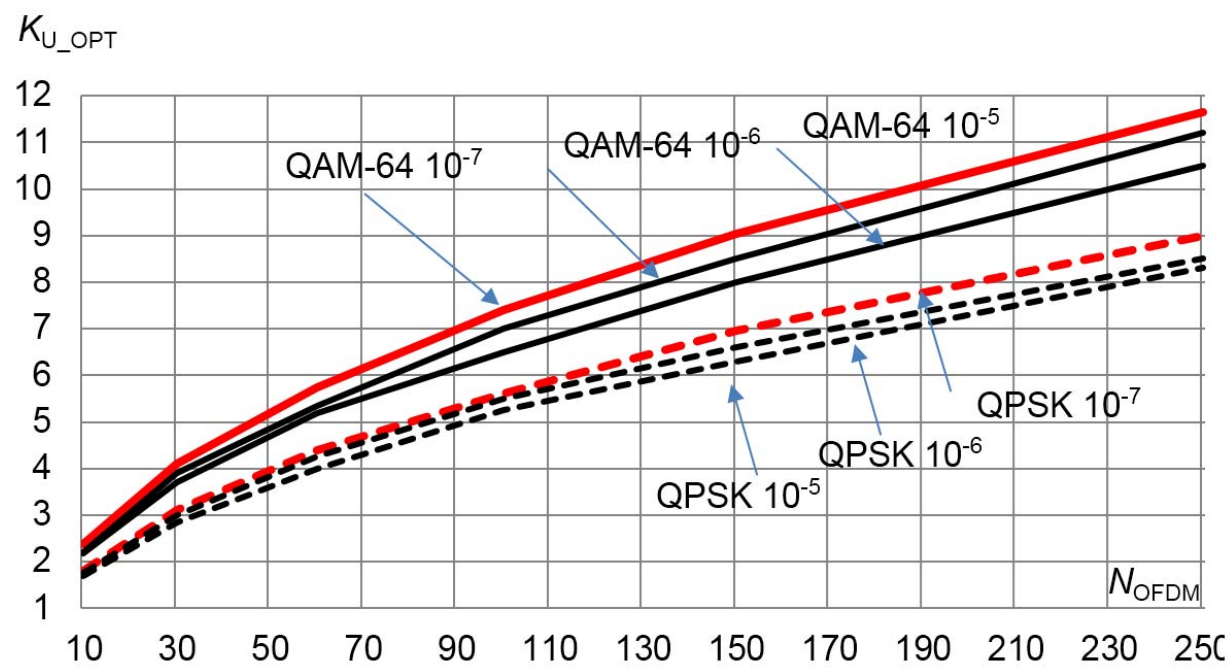

Figure 10. $K_{\mathrm{U}_{-} \text {OPT }}$ for MPK types QAM-64 and QPSK with different requirements for information reliability transmission

The analysis of function $\frac{K_{\mathrm{P}_{\mathrm{O}} \text { OPT }}}{N_{\mathrm{OFDM}}}=f\left(N_{\mathrm{OFDM}}, k\right)$ (Fig. 11) shows that for the case of $k=6$ (QAM-64) and $p_{\mathrm{b}_{-} 0}=10^{-6}$ :

$\left.\frac{K_{\mathrm{P}_{\text {_OPT }}}}{N_{\text {OFDM }}}\right|_{\text {MAX }}=f\left(N_{\text {OFDM }} ; k ; p_{\mathrm{b}_{-} 0}\right)=f\left(k ; p_{\mathrm{b}_{-} 0}\right)=f\left(6 ; 10^{-6}\right)=0.49$,

so the ratio of optimal OFDM signal gain factor $K_{\mathrm{P}_{-} \text {OPT }}$ to subcarriers number $N_{\text {OFDM }}$ is an invariant value (for specific MPK type and information reliability level).

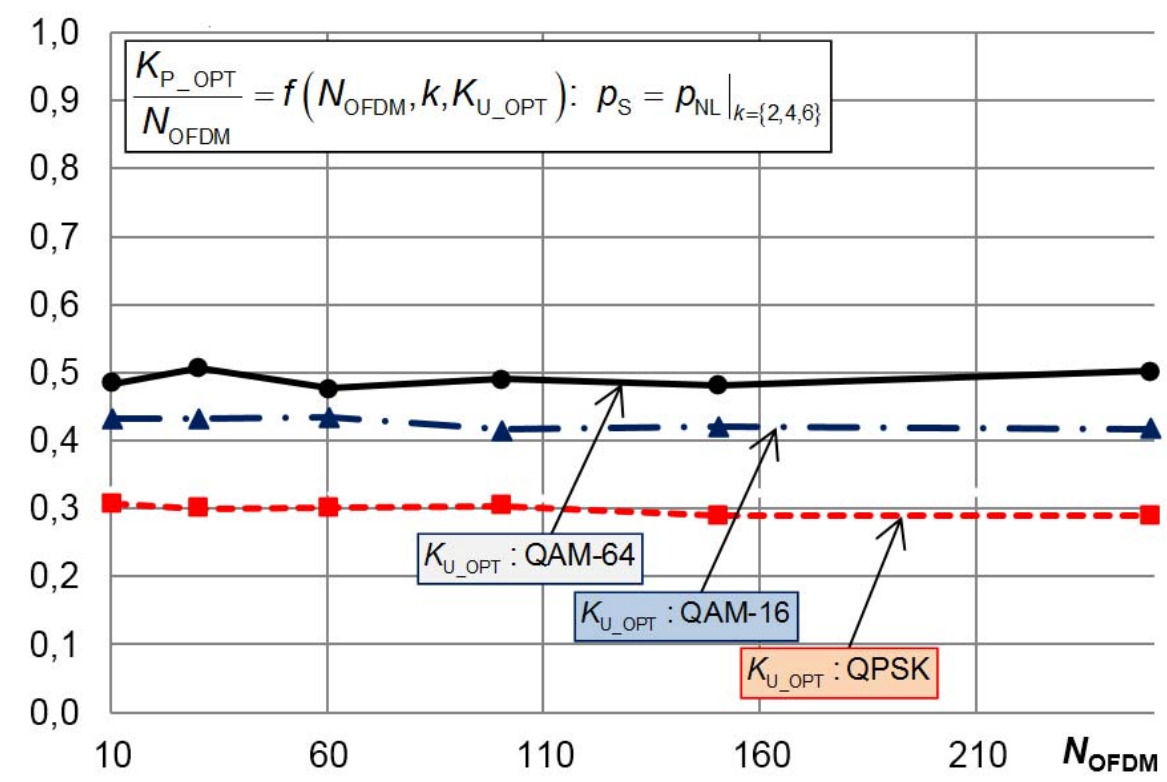

Figure 11. The ratio of optimal OFDM signal gain factor $K_{\mathrm{P}_{-} \text {OPT }}$ to subcarriers number $N_{\text {OFDM }}$

The results given in the same way for different MPK types and different reliability requirements (BER) are shown in Table 1; the same independency from $N_{\text {OFDM }}$ was found for a rate $K_{\text {P_opT }} / N_{\text {OFDM }}$. 
Table 1. The ratio values $K_{\mathrm{P}_{-} \mathrm{OPT}} / N_{\mathrm{OFDM}}=f\left(N_{\mathrm{OFDM}} ; k ; p_{\mathrm{b} \_0}\right)$

\begin{tabular}{|l|l|l|l|}
\hline MPK $\backslash$ BER & $\boldsymbol{p}_{\mathbf{b} \_\mathbf{0}}<\mathbf{1 0}^{-\mathbf{5}}$ & $\boldsymbol{p}_{\mathbf{b} \_\mathbf{0}}<\mathbf{1 0}^{\mathbf{- 6}}$ & $\boldsymbol{p}_{\mathbf{b} \_\mathbf{0}}<\mathbf{1 0}^{-\mathbf{7}}$ \\
\hline QPSK & 0,27 & 0,30 & 0,32 \\
\hline QAM-16 & 0,38 & 0,43 & 0,47 \\
\hline QAM-64 & 0,44 & 0,49 & 0,54 \\
\hline
\end{tabular}

It is important to highlight although the OFDM transmission mode is significantly worse than SC transmission mode by energy characteristics, but it is possible potentially to compensate for lost information reliability in OFDM by using antinoise codes methods in structures of signal-code sequences with specifically chosen MPK type and antinoise code method.

\section{Conclusion}

The efficiency of using an optimal OFDM aggregate signal gain factor is shown in this work based on the results of simulation modelling. In this case it is possible to reach the independency of energy parameters and OFDM signal information reliability from subcarriers number. It is also shown how exactly an optimal gain factor depends on OFDM subcarriers number.

Analytically the condition of achievement an optimal OFDM signal gain factor is based on solving an integral equation where a form of amplitudes distribution density function in defined by condition (8) with distribution parameter (6b) when the voltage level value and SER value are known, and the value of optimal OFDM signal value gain is sought-for in this case.

The presented results of the optimal OFDM signal gain values are given based on a statistical modelling. Specific OFDM signal gain values provide a possibility to calculate an appropriate energy parameter value (12) which is a base for further analytical calculation of SER value, BER value and probability of non-linear distortions in an amplifier on the transmitting side.

The substantiated effect of the described method of forming an aggregate OFDM signal provides an opportunity to use this method as a tool of multistation access in such perspective technologies as LTE and other similar technologies. In addition to this, the described method for amplification an OFDM signal presents the OFDM technique as a potentially efficient technique in communication channels with multipath transmission, because changing OFDM subcarriers number is possible without any influence on direct OFDM signal beam energy. In turn, changing OFDM subcarriers number makes it possible to manage the length of OFDM signal period against beams lagging, that decreases an impact of multipath factor on OFDM signal receiving quality and reliability.

The losses that are given due to an OFDM signal usage instead of SC transmission can be compensated by selecting an appropriate signal-code sequence that includes a corresponding MPK type and antinoise code with proper parameters to bring the information reliability up to some required level.

\section{References}

1. Chang, R.W. (1966) Orthogonal Frequency Division Multiplexing. U.S. Patent 3388455.

2. Prasad, R. (2004) OFDM for Wireless Communications systems. Artech House Publishers.

3. Ghosh, A., Zhang, J., Andrews, J.G., and Muhamed, R. (2004) Fundamentals of LTE. Prentice Hall.

4. Gangwar, A., Bhardwaj, M. (2012) An Overview: Peak to Average Power Ratio in OFDM system \& its Effect. International Journal of Communication and Computer Technologies, Volume 01, Number 2, pp. 22-25.

5. Uryvsky, L. and Osypchuk, S. (2013) OFDM signal energy characteristics research in channel with permanent parameters. Telecommunication Sciences, Volume 4, Number 2, pp. 22-27. 